\title{
Testing discrepancies in the measurement of the acceleration of gravity in a physical pendulum experiment
}

\author{
Ana R. Romero Castellanos ${ }^{1}$, C. E. Alvarez-Salazar ${ }^{1}$, P. C. de Holanda*1@ \\ ${ }^{1}$ Universidade Estadual de Campinas, Instituto de Fíísica Gleb Wataghin, Campinas, SP, Brasil
}

Received on June 26, 2019. Revised on September 24, 2019. Accepted on October 9, 2019.

\begin{abstract}
After analysing results on the measurement of the acceleration of gravity using a physical pendulum in our teaching laboratory, we found that the value reported by the students is always higher than the theoretical expectations. In this article we analyze critically some of the students hypotheses for such displacement, and we perform an investigation of our own to detect what is leading to this result. We disregarded any flaw on the experimental procedure performed by the students, and instead, concluded that the miscalibration of the laboratory instruments was distorting the students measurements, leading to a $\sim 5 \%$ increase in the calculated value of the acceleration of gravity.
\end{abstract}

Keywords: Physical Pendulum, acceleration of gravity

\section{Introduction}

The analysis of the oscillatory behaviour of physical systems is a fundamental part of physics courses at the undergraduate level, where simple harmonic oscillators are studied, both from the theoretical and the experimental point of view.

A very common experiment in fundamental physics courses, which has been used throughout the years, consists on the analysis of the oscillatory motion of a physical pendulum [1] 3 , which is generally used to get a measurement of the gravitational acceleration $g$. The main advantages of this experiment are its simplicity, low cost and wealth of both physical and mathematical concepts.

From the theoretical physics point of view, the physical pendulum can be used to understand the behavior of systems acted on restoring forces proportional to the displacement from the equilibrium point, the concept of a rigid body and the determination of its center of mass (CM) and moment of inertia (and the radius of gyration), the use of Steiner's theorem, the effect of damping, among others 4].

The mathematical techniques required to analyze the oscillatory motion of the physical pendulum require the use of Taylor series to approximate a second order nonlinear differential equation to a linear one and the solution of differential equations with constant coefficients using characteristic equations, leading to the transformation of complex exponentials to real-valued functions.

Finally, from the experimental physicist perspective, the physical pendulum requires to configure the experiment in order to test the validity of the theoretical

* Correspondence email address: holanda@ifi.unicamp.br approximation, data taking skills and processing, the identification of diverse sources of uncertainty, the ability to choose appropriately the independent and dependent variables to make a plot showing their relationship, the statistical analysis required to fit a model to the data, and the ability to evaluate its reliability (which could lead to the observation of faults in the previous steps).

For some years now, students at our university have been performing the physical pendulum experiment with some success. The rigid body consists of a metallic stick of length $L$ through which holes have been drilled at various positions, in such a way that the rod can be hanged from different points, changing the distance between the CM and the pivot point. A variation is obtained when a smaller metallic bar is attached (at different points) to the stick, modifying the position of the CM and the moment of inertia.

The full dynamical equation for the physical pendulum without energy dissipation can be written as:

$$
I \frac{d^{2} \theta}{d t^{2}}+M g D \sin \theta=0
$$

where $\theta$ is the angular displacement from the vertical, $I$ is the moment of inertia about a pivot point placed at distance $D$ from the Center of Mass - CM, $M$ is the pendulum mass and $g$ the gravity acceleration. With the small angle approximation, $\sin \theta \approx \theta$, the above equation describes a harmonic oscillator with period $T$ given by:

$$
T=2 \pi \sqrt{\frac{I}{M g D}},
$$

As stated previously, students obtain the acceleration of gravity $g$ through a linear relationship between the 
compound variables $T^{2} D$ and $D^{2}$, as will be shown in section 3

We have noticed that the value of the acceleration of gravity $g$ measured by the students, although with a wide distribution, consistently gives a higher number than the expected value at our position on Earth ${ }^{1} g=9.7861$ $\mathrm{m} / \mathrm{s}^{2}$. For instance, using the data taken by fifty groups of students, shown in figure 1 , the average value measured is $g=10.34 \pm 0.02 \mathrm{~m} / \mathrm{s}^{2}$.

In order to explain this discrepancy between their result and the known value of $g$, students give all kind of explanations: non-validity of the small angle approximation in their experiment, the use of a non-uniform rod (due to the holes drilled on it), the difficulty in finding the exact position of the CM, the presence of air currents in the laboratory producing unexpected effects, the oscillations of the rod not happening in a plane, to mention just a few.

We present here our own investigation of the oscillatory motion of the physical pendulum, trying to figure out why such discrepancies appear in our experimental setup.

This article is organized as follows: in Sec. 2 we present the experimental setup used, discarding directly some of the explanations argued by the students. In Sec. 3 we present the results obtained when the same analysis performed by the students is applied to a data set collected under the same experimental conditions present in the classroom. In Sec. 4 we present different hypotheses raised and tested to explain the discrepancy in the measurement of $g$. In section 5. once we have solved the problem with the acceleration of gravity, we take advantage of the experiment to analyze the variation of the radius of gyration of the physical pendulum. Finally, we present our conclusions in Sec. 6.

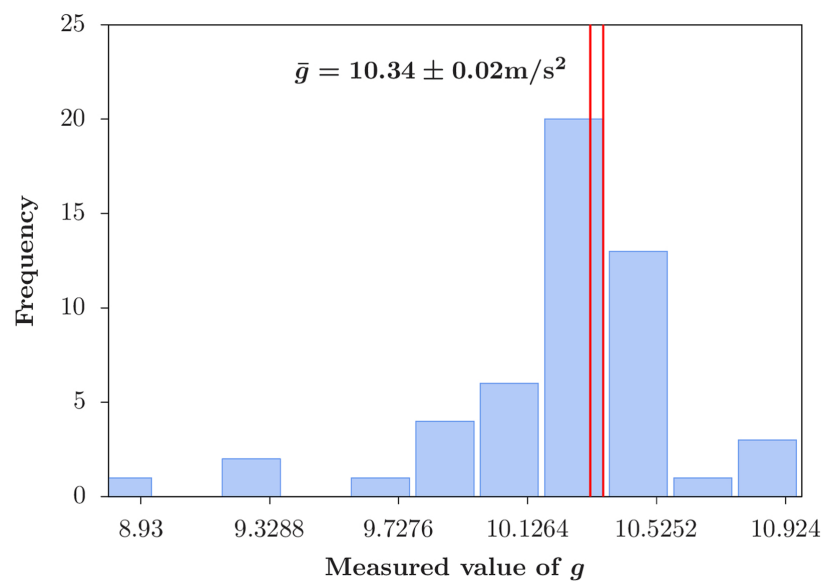

Figure 1: Distribution of the results for the measurement of the acceleration of gravity $g$ by a sample of fifty groups. The weighted average of the results is shown in the graph, with its boundaries indicated by the red vertical lines.

${ }^{1}$ This value was obtained using the Gravity Information System of PTB

\section{Experimental setup}

The experiment proposed to the students consists on measuring the period of oscillations of an aluminum stick of length $L=1.4830 \pm 0.0005 \mathrm{~m}$ and mass $M=0.9216 \pm$ $0.0001 \mathrm{~kg}$, through which circular holes, separated by a distance of approximately $5 \mathrm{~cm}$, have been drilled. Using these holes, the rod can be hanged from a pivot, changing the distance between the $\mathrm{CM}$ and this point. Using an iron bar of length $l=0.1600 \pm 0.0005 \mathrm{~m}$ and mass $m=0.3760 \pm 0.0001 \mathrm{~kg}$, it its possible to change the position of the $\mathrm{CM}$ and the moment of inertia of the system, placing the bar at different positions along the rod.

The bar is pivoted from the holes using a screw in a clamp fixed to the stand, and in order to keep the oscillations of the bar in a plane, a nut is fastened to the screw. The nut is fastened in such a way that keeps the stick fix, but free to oscillate in a plane. Figure 2 shows a schematic representation of the experimental setup. This configuration, alongside with the fact that the experiment is performed in a closed lab, discards immediately two of the possible explanations given by the students to justify the high value of $g$ they report, namely, the air currents in the lab and the oscillation not happening in a plane.

Releasing the bar from angles less than $15^{\circ}$, students let the system perform 20 oscillations, and use a photogate system in pendulum mode in order to measure the

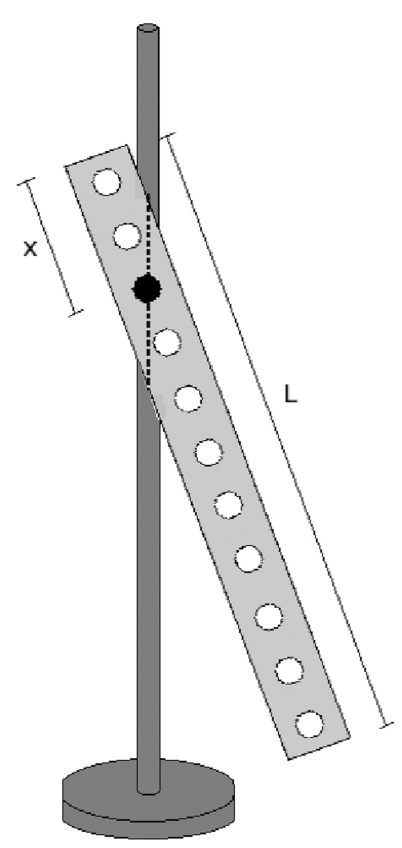

Figure 2: Schematic representation of the experimental setup used by the students to describe the oscillatory motion of a physical pendulum. In order to change the distance between the pivot point and the center of mass, the stick is hung from different holes, drilled at variable distances $x$ from the upper end. 
period of oscillations. Taking this into consideration, the oscillations of the pendulum should be consistent with the small angle approximation.

Even though this configuration discards deviations of the small angle approximation as a source of discrepancy in the measured value of the acceleration of gravity, we made our own analysis of this and other hypotheses, which we present in Sec. 4

\section{Statistical analysis usually performed by the students}

In order to analyze the result of their experiments, consisting on sets of 20 measurements of the period of oscillation for each pivot point, students find the mean and standard deviation for each period, write the moment of inertia about the pivot using the parallel-axis or Steiner's theorem ${ }^{2}, I=M k^{2}+M D^{2}$, where $k$ is the radius of gyration, and manipulate equation (2), in order to find a linear relationship between the compound variables $T^{2} D$ and $D^{2}$,

$$
D^{2}=\frac{g}{4 \pi^{2}} T^{2} D-k^{2},
$$

in such a way that the values of the acceleration of gravity and the radius of gyration could be obtained from the slope and y-intercept, respectively. Note that we have written equation (3) in a form where the variable with higher relative uncertainty $\left(D^{2}\right)$ appears as the dependent variable. This is due to the high precision of the photogate used to measure the period of oscillation, leading to a relative uncertainty in the compound variable $T^{2} D$ of the order $0.01 \%$, much lower than the corresponding to $D^{2}$, of the order $2 \%$.

Using the aluminum stick only, we performed the experiment and the data analysis under the same conditions used by the students, taking the center of mass of the stick at $L / 2$, finding an acceleration of gravity $g=10.34 \pm 0.08 \mathrm{~m} / \mathrm{s}^{2}$ and a radius of gyration $k^{2}=0.184 \pm 0.002 \mathrm{~m}^{2}$.

The result for the radius of gyration is consistent with the expected value $k^{2}=L^{2} / 12=0.1833 \pm 0.0001 \mathrm{~m}^{2}$, but the value of the acceleration of gravity has a $5.66 \%$ deviation compared to the known value. In the following section we will set the students (and our) hypotheses to test, in order to find the source of this discrepancy.

\section{Hypotheses for the deviation of $g$}

\subsection{Deviation of the small angle approximation}

Usually, students are suggested to use the small angle approximation and replace $\sin \theta \approx \theta$, from which the period of oscillations in Eq. (2) is directly obtained, and leading to an analytic solution of the evolution equation.

\footnotetext{
${ }^{2}$ In order to help students understand the concepts of moment of inertia and the parallel-axis theorem, it could be useful to calculate the first one without using integrals, as in reference [5], and perform an experimental demonstration of the second, as shown in 6 .
}

This is a reasonably good approximation, even though it has a non-negligible influence on the final result. Since the approximation overestimates the restoring torque on the rod, it is expected that the measured period would increase with the initial angle $\theta_{0}$ from which the pendulum is set to motion in comparison with the asymptotic value when $\theta_{0} \rightarrow 0$. Then, using Eq. (2) would lead to a lower value for $g$ than expected. These considerations are enough to discard the effect as an explanation to the discrepancy we are considering, and may even enhance it. So it is worthwhile to analyze it quantitatively in more detail.

To investigate how the approximation distorts the final result, we numerically solved the full equation, without any approximation, and calculated the pendulum period $T$. We found out that the function $f\left(\theta_{0}\right)$ relating the measured period and the asymptotic period values depends on the pendulum initial angular displacement $\theta_{0}$, but is independent of $\mathrm{D}$ and $\mathrm{k}$, and we can write:

$$
T_{\text {measured }}=f\left(\theta_{0}\right) \times T_{\text {asymptotic }}
$$

where $f\left(\theta_{0}\right)$ is determined numerically and $T_{\text {asymptotic }}$ is the asymptotic limit when $\theta_{0} \rightarrow 0$. Since eq. (3) refers to $T_{\text {asymptotic }}$, it can be written in terms of $T_{\text {measured }}$ with the modification:

$$
g \rightarrow \frac{g}{f\left(\theta_{0}\right)^{2}}
$$

Then, the standard analysis using small angle approximations, compound variables and linear regression of Eq. (2), would lead to no modification on the determination of $k$, and to a lower value of $g$ by a factor $f\left(\theta_{0}\right)^{2}$, which corresponds to a correction factor $\left(1-1 / f\left(\theta_{0}\right)^{2}\right) \%$. For most of the students setups we expect an initial angle up to $\theta_{0}=20^{\circ}$, which leads to corrections of the order of $1-2 \%$, as can be seen in Fig. 3 .

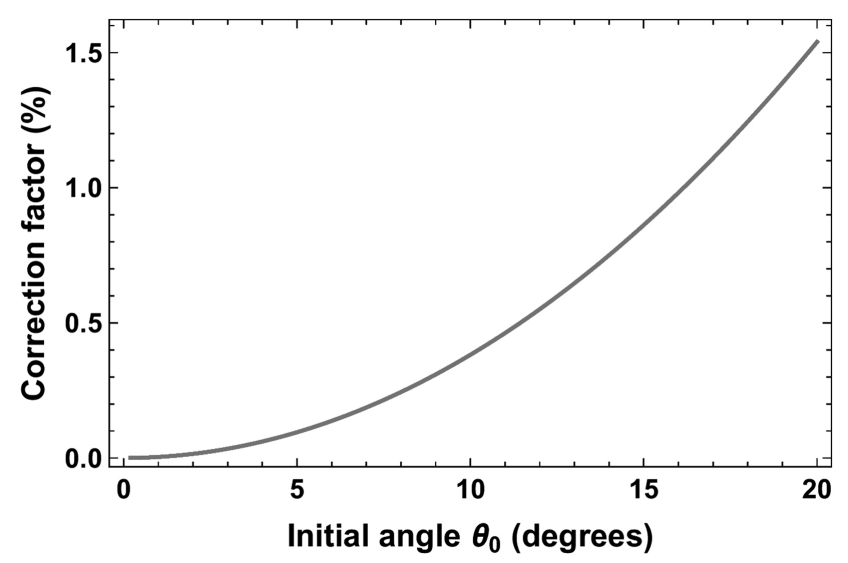

Figure 3: Correction factor $\left(1-1 / f\left(\theta_{0}\right)^{2}\right) \%$ for the acceleration of gravity due to disregard of the correction from small angle approximation 


\subsection{Consideration of the screw-nut system}

Another possible explanation points to the lack of consideration of the screw-nut system, which is not usually taken into account in the analysis. The screw-nut system is responsible for keeping the oscillations of the aluminum stick on a plane. However, since the screw and the nut rotate alongside the stick, the total moment of inertia changes compared to the stick alone. Besides, the CM position of the whole system also changes depending on the pivot point position.

As the screw is inside the clamp and is impossible to take it out, the best we could do was to estimate the mass $\mu$ of the screw-nut system, and take it into account on our theoretical predictions. We use the value $\mu=45 \pm 5 \mathrm{~g}$ for the screw-nut system mass. In this section we analyze both effects and the influence they have in the final experimental results.

The CM of the system composed by the aluminum stick, of mass $M$ and length $L$, and the screw-nut, which we consider as a particle of mass $\mu$ placed at different positions $x$ along the rod (measured from the upper end of the stick), as shown in figure 2, is given by

$$
X_{\mathrm{CM}}=\frac{M \bar{x}_{\mathrm{CM}}+\mu x}{M+\mu},
$$

where $\bar{x}_{\mathrm{CM}}$ is the CM position of the aluminium bar alone, without the screw-nut unit. The distance between the axis of rotation and the CM becomes

$$
D=\left|X_{\mathrm{CM}}-x\right|=\left|\frac{M}{M+\mu}\left(\bar{x}_{\mathrm{CM}}-x\right)\right|=\frac{1}{1+\mu / M} D_{s},
$$

where $D_{s}=\left|\bar{x}_{\mathrm{CM}}-x\right|$ is the quantity measured by the students, assuming a fixed position for the CM. The true CM position is slightly closer to the upper end of the stick, which we use as the origin for the measurement of the coordinate $x$. We show in the left pannel of Fig. 4 the CM displacement due to the changing position of the screw-nut unit, which can be as high as $5 \%$ for our experimental setup.

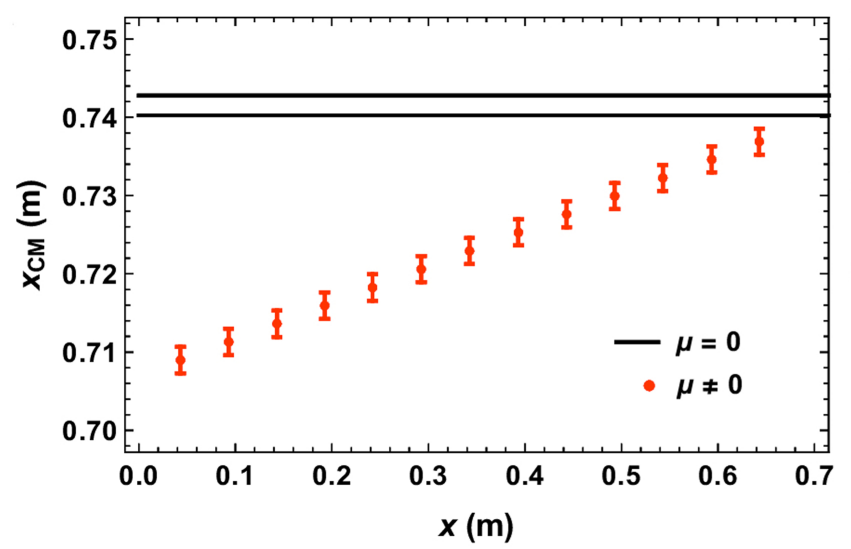

The second modification comes from the change in the moment of inertia of the system. Assuming that the screw-nut system has a total radius of gyration $k_{a}$, we can write the following expression:

$$
I=M\left(k_{s}^{2}+D_{s}^{2}\right)+\mu k_{a}^{2}=(M+\mu)\left(D^{2}+k^{2}\right)
$$

where $k_{s}$ is the radius of gyration of the system composed by the stick alone, without the screw-nut unit. Replacing eq. (4) in eq. (6) we get:

$$
k^{2}=\frac{\mu / M}{(1+\mu / M)^{2}} D_{s}^{2}+k_{s}^{2} \frac{1}{1+\mu / M}\left(1+\frac{\mu k_{a}^{2}}{M k^{2}}\right)(7)
$$

The modifications on the system radius of gyration can be as high as $3 \%$, as can be seen in the right pannel of Fig. 4.

The relation (3) between $D^{2}$ and $T^{2} D$ becomes nonlinear, since the radius of gyration $k$ depends on $D$, and we cannot use linear regression to extract the value of $g$. However, we can rewrite eq. (3) in a more convenient way. Replacing eq. (5) and eq. (7) in eq. (3) we get after some algebraic manipulation:

$$
D_{s}^{2}=\frac{g}{4 \pi^{2}} T^{2} D_{s}-k_{s}^{2}\left(1+\frac{\mu k_{a}^{2}}{M k^{2}}\right) .
$$

With this relation we recover the linearity from which we can extract $g$. Therefore we conclude that the only effect of disconsidering the screw-nut unit is to extract a slightly different value for the radius of gyration of the system. However, the effect is negligible for the physical configuration used.

\subsection{A problem with the photogate measurements}

Another source of discrepancy could come from problems with the time measurements obtained with the photogate.

In order to set this hypothesis to test, we performed two modifications in the experimental setup.

First, instead of measuring the period of oscillations making the full wide of the stick $(3.80 \pm 0.05 \mathrm{~cm}$ wide)

Figure 4: Left pannel: difference between the position of the $\mathrm{CM}$ for the aluminum stick (black lines, including the uncertainty in the measurement), and the center of mass (red dots) calculated using Eq. (4). Right pannel: same for the radius of gyration and Eq. (7). 
cross the photogate (the same configuration used by the students), we placed a little stick of negligible mass (approximately $2 \mathrm{~g}$ ) in the center of the aluminum one. These configuration, and the results obtained for the measurement of 20 oscillations, are shown in Fig. 5

From this figure, we can see that the mean periods are inconsistent, maybe because of the time the stick stops the light beam of the photogate. In this case, a greater period of oscillation could lead to a difference in the slope in Eq. (3), changing the value of the acceleration of gravity.

Encouraged with this possibility, and with the new configuration for the measurements, we performed again the experiment and data analysis, finding an acceleration of gravity $g=10.09 \pm 0.06 \mathrm{~m} / \mathrm{s}^{2}$, which certainly corresponds to a decrease in the previous value, $10.34 \pm$ $0.02 \mathrm{~m} / \mathrm{s}^{2}$, but not enough to correct the result of the experiment.

For the second modification, instead of measuring the oscillation period with the photogate in pendulum mode, where each oscillation period is measured individually, we measured 20 oscillation periods with the use of a digital chronometer. We obtained a systematic decrease of the average period time around $3 \%$ in comparison with the photogate one. The associated error increased by a factor of $\sim 2$, with the decrease in the equipment precision being partly compensated by a continuous measurement of a large number of periods (anyway the uncertainties in the period does not affect the final uncertainties, due to the absence of $T$ in the dependent variable chosen).

Using this set of data, we obtained for the gravity acceleration the value $g=9.70 \pm 0.07$, leading us to the conclude that the photogate miscalibration were the main cause of error that lead to the systematic displacement of the value of gravity acceleration measured by the students. Including in our analysis a correction discussed in section 4.1 of $0.4 \%$, compatible with an initial angle $\theta_{0}=10^{\circ}$, we obtain the final value of our measurement

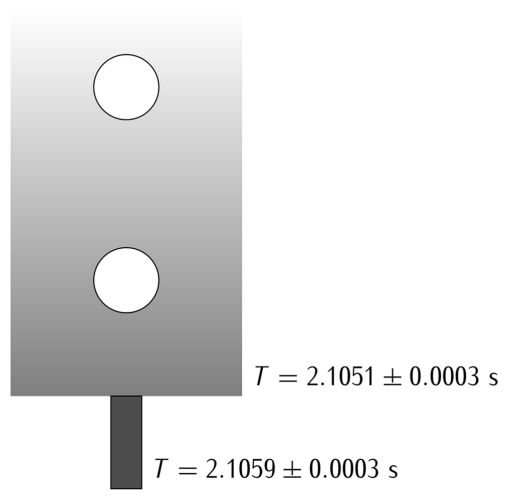

Figure 5: Comparison of the measurements performed with the photogate for the period of oscillation about the same point, as measured using the aluminum stick and a negligible mass object attached to it. to be:

$$
g=9.74 \pm 0.08 \quad(1 \sigma)
$$

in accordance with the theoretical value.

The previous analysis show how little deviations in the photogate system calibration can affect dramatically the acceleration of gravity result measured in the experiment, while properties intrinsic to the rigid body, such as its radius of gyration, are still measured with reliability.

\section{Modifying the radius of gyration}

As the result on the acceleration of gravity always leaves students puzzled, they miss the opportunity to understand the concept of the radius of gyration of a body, and how it changes depending on its mass distribution. Taking this into account, and the fact that the concept of radius of gyration is a key property of a rigid body 7,8 that can be difficult to understand for students, we used the iron bar described in Sec. 2 in order to change the moment of inertia of the system.

With this new configuration, we got data for the period of oscillation as a function of the distance from the pivot to the center of mass, shown in Fig. 7. In the left panel of this figure, we can see that as the position of the bar is closer to the center of the stick, what makes the position of the CM closer to its upper end, the pendulum takes a shorter time in order to perform a complete oscillation, due to the fact that the radius of gyration of the rigid body is decreasing.

From Eq. (3) it is possible to show that the minimum period of oscillation of the physical pendulum occurs when the distance from the CM to the pivot is equal to the radius of gyration, and we have marked (in black) these points for each curve in the right panel of Fig. 7. where a comparison of the period of oscillation of the physical and simple pendulums have been made. From this graph, we can see that as the distance from the CM to the pivot increases the period of the physical pendulum becomes closer to the period of a simple pendulum, as in this case the geometrical extension and the mass distribution of the rigid body plays a very small role on the period of oscillation, as the body could be described as a particle with all its mass concentrated on the CM.

In order to understand this, Fig. 7] shows that changing the position of the center of mass, as a consequence of different positioning of the iron bar, changes the value

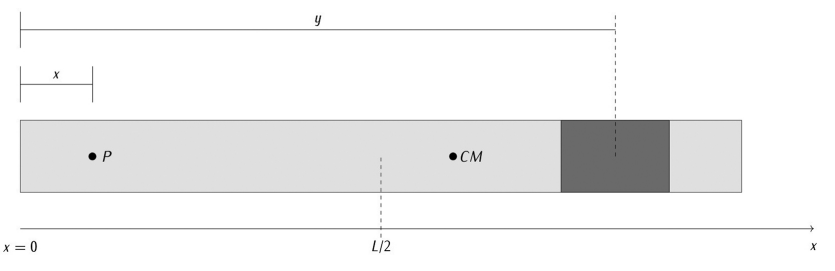

Figure 6: Diagram illustrating the geometry of the system in order to calculate the radius of gyration. 

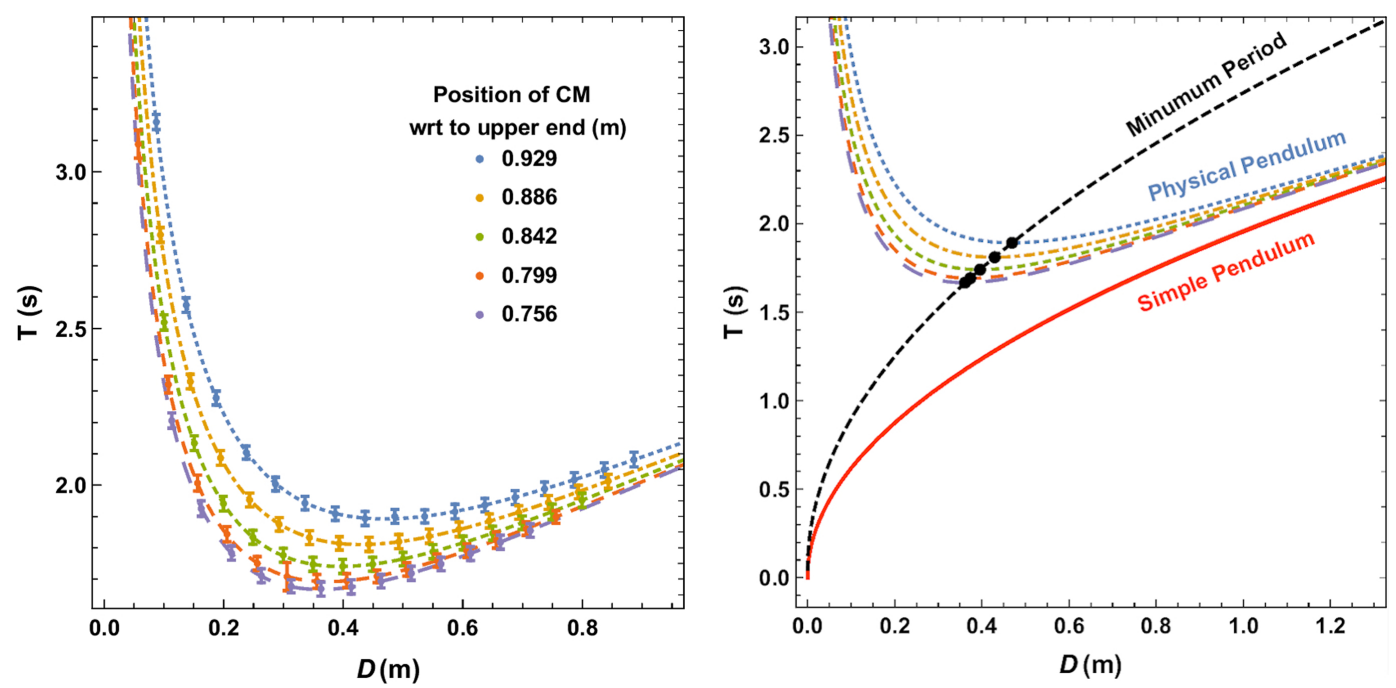

Figure 7: Period of oscillation of a physical pendulum composed by the aluminum stick and an iron bar. The left panel shows the experimental results when the bar is placed on different positions along the stick, where data sets are labelled by the position of the $\mathrm{CM}$ (in $\mathrm{m}$ ), measured from the upper end of the stick. The right panel shows a comparison of the experimental results (colored lines) obtained with the physical pendulum and the period of a simple pendulum of length $D$ (red line). The dashed line (black) shows a fit for the minimum period of oscillation of the different pendulums, obtained when the distance between the pivot and the CM is equal to the radius of gyration.

of the radius of gyration of the system, which can be read as the $D$ coordinate giving the minimum period for each curve. Using Steiner's theorem, and the definition of radius of gyration, we can write:

$$
I_{P}=I_{C M}+(M+m) D^{2}
$$

where $I_{P}$ is the moment of the total system with respect to the pivot point, and $D=x_{C M}-x$, (see figure (6) where the position of center mass $x_{C M}$ is given by

$$
x_{C M}=\frac{M L / 2+m y}{M+m} .
$$

we used Steiner's theorem in order to obtain $I_{P}$

$$
I_{P}=\frac{1}{12} M L^{2}+M\left(\frac{L}{2}-x\right)^{2}+\frac{1}{12} m l^{2}+m(y-x)^{2} .
$$

It can be shown that the radius of gyration of the system, when the iron bar of mass $m$ is at position $y$ from the upper end of the stick, is given by

$$
k^{2}=\frac{1}{12(M+m)}\left(M L^{2}+m l^{2}\right)+\frac{m M}{(M+m)^{2}}\left(y-\frac{L}{2}\right)^{2},
$$

from which we can see, as expected, that $k$ depends only on intrinsic properties of the rigid body, its mass distribution and geometrical characteristics, but is independent of the position of the pivot ( $x$ in the notation of subsection 4.2 or the distance from the CM to the pivot (D).

From eq. 10 we can see that, as the iron bar gets closer to the center of mass of the aluminum stick $(y \rightarrow L / 2)$ (corresponding to a lower value for the position of the CM in Fig. 7), the radius of gyration decreases (the minimum of the period of oscillation in Fig. 7 moves to the left), and $k$ becomes

$$
k^{2}=\frac{M}{M+m} k_{M}^{2}+\frac{m}{M+m} k_{m}^{2}
$$

where $k_{M}$ and $k_{m}$ are the radii of gyration of the aluminum stick and the iron bar, respectively.

\section{Conclusions}

Through our investigation, we identified two sources for the discrepancies on the value of the acceleration of gravity $g$ measured through the years in our teaching labs.

First, the small angle approximation can lead to slightly lower values for $g$ if we set the initial angle for values higher than $\sim 10^{\circ}$. However, most of the students do not exceed this value, and the discrepancy we noted point to the opposite direction, increasing the value of $g$ in comparison with its expected value.

Second, we identified a problem with the calibration of the photogates used. This miscalibration leads to a systematic displacement of the central value for the measurement of the acceleration of gravity, affecting all the experimental setups that used these photogates. Besides, the uncertainty associated with time measurements using the photogate were critically undervalued in the student analysis. Although such uncertainty does not have an effect on the statistical analysis we used to determined $g$, it should be taken into account in a more detailed statistical treatment. 
It is important to note that our analysis did not give a final solution to the discrepancy in the measurement of the acceleration of gravity, in the sense that only a careful calibration of the photogates, performed alongside with the experiment, could improve the results and give the correct uncertainties associated with the experiment.

In our opinion, a more critical consequence from the problem we identified would be on the students formation. All experimental courses give the appropriate emphasis on the necessity of correctly treating uncertainties and propagating errors in any experimental procedure. When the measurement of a known quantity gives a value systematically outside the confidence region defined by the experimental uncertainties, we have to know what is causing such effect, in the risk of undermining the importance of uncertainties for the students. So the lesson we took from this is: always calibrate the measurement equipment with the students!

\section{Acknowledgements}

This study was financed in part by CAPES, finance code 001 (A.R.R.C), and CNPq under grant number 159237/2015-7 (C.E.A.S). The authors also acknowledge the support of FAPESP funding grant 2014/19164-6.

\section{References}

[1] H. Weltin, American Journal of Physics 32, 267 (1964).

[2] G.O. Kolodiy, The Physics Teacher 17, 52 (1979).

[3] T.H. Richardson and S.A. Brittle, Physics Education 47, 537 (2012).

[4] G.L. Baker and J.A. Blackburn, The pendulum: a case study in physics (Oxford University Press, Oxford, 2005).

[5] B. Oostra, The Physics Teacher 44, 283 (2006).

[6] B. Denardo, The Physics Teacher 36, 56 (1998).

[7] G.B. Russeva, G.G. Tsutsumanova and S C Russev, Physics Education 45, 58 (2010).

[8] M. Kladivová and L. Mucha, European Journal of Physics 35, 025018 (2014). 